\title{
The Role of Control Measures and the Environment in the Transmission Dynamics of Cholera
}

\author{
Nicholas Kwasi-Do Ohene Opoku $\mathbb{D D}^{1,2}$ and Cecilia Afriyie ${ }^{1,3}$ \\ ${ }^{1}$ African Institute for Mathematical Sciences, Accra, Ghana \\ ${ }^{2}$ University of Cape Coast, Cape Coast, Ghana \\ ${ }^{3}$ Kwame Nkrumah University of Science and Technology, Kumasi, Ghana \\ Correspondence should be addressed to Nicholas Kwasi-Do Ohene Opoku; nicholas@aims.edu.gh
}

Received 4 August 2019; Revised 13 November 2019; Accepted 26 December 2019; Published 3 February 2020

Academic Editor: Sergei Grudsky

Copyright (C) 2020 Nicholas Kwasi-Do Ohene Opoku and Cecilia Afriyie. This is an open access article distributed under the Creative Commons Attribution License, which permits unrestricted use, distribution, and reproduction in any medium, provided the original work is properly cited.

\begin{abstract}
Cholera is an infectious intestinal disease which occurs as a result of poor sanitation and lack of basic education in its transmission. It is characterized by profuse vomiting and severe diarrhea when an individual eats food or drinks water contaminated with the Vibrio cholerae. A dynamic mathematical model that explicitly simulates the transmission mechanism of cholera by taking into account the role of control measures and the environment in the transmission of the disease is developed. The model comprises two populations: the human population and bacteria population. The next-generation method is used to compute the basic reproduction number, $R_{0}$. Both the disease-free and endemic equilibria are shown to be locally and globally stable for $R_{0}$ values less than unity and unstable otherwise. Necessary conditions of the optimal control problem were analyzed using Pontryagin's maximum principle with control measures such as educational campaign and treatment of water bodies used to optimize the objective function. Numerical values of model parameters were estimated using the nonlinear least square method. The model simulations confirm the significant role played by control measures (education and treatment of water bodies) and the bacteria in the environment in the transmission dynamics as well as reducing the spread of cholera.
\end{abstract}

\section{Introduction}

Cholera is an infectious intestinal disease characterized by profuse vomiting and acute watery diarrhea which is caused by eating food or drinking water contaminated with a bacterium called Vibrio cholerae $[1,2]$. When an individual is infected with cholera, it leads to dehydration and if untreated, can result to death. For centuries, cholera has remained one of the most dreadful diseases worldwide. Vibrio cholerae, the causative organism for cholera, is a Gram-negative comma-shaped bacterium. It has flagellum for motility and pili (a hairlike structure which it uses to attach tissues). Brackish water or saltwater serves as its natural habitat; as a result, it is commonly found in oysters and shellfish.

Cholera was first described by Hippocrates who is considered as one of the most outstanding figures in the history of medicine. Historically, the Ganges Delta region in Asia is considered the first region to be infested with cholera [3]. It is believed that cholera spread throughout the world from this region. Several epidemics occurred in Asia during the fifteenth and eighteenth centuries. In the year 1892, a major cholera outbreak occurred in Germany which killed over 10,000 people. It was found to have been caused by a defect in the German waste removal system. Over the centuries, seven major pandemics of cholera had occurred since 1817, that is, during the nineteenth and twentieth centuries [3, 4]. The seventh pandemic began in Indonesia in the year 1961 which reached West Africa in 1970 and also in America in the year 1991. During the nineteenth century, cholera was not only considered a terrifying disease but was also a challenge to national identity and national economy [5].

There is a general burden of cholera on human population. Cholera serves as an indicator to lack of social 
amenities in most countries, especially developing countries, which is a global threat to public health. The populations living in unsanitary conditions are increasing, and the reemergence of cholera has also crept in. The number of cholera outbreaks reported by the World Health Organization (WHO) continues to increase among which includes a total of 838,315 from 2004 to 2008 , as compared to 676,651 cases notified from 2000 to 2004 [6]. Also in 2006, 52 countries reported 236,896 cholera cases including 6311 deaths with a case-fatality ratio of $2.7 \%$ [7]. Again, in the year 2011, a total of 589,854 cholera cases including 7,816 cholera-induced deaths were reported worldwide from 58 countries with a case-fatality ratio of $1.3 \%$ [8].

Multiple outbreaks continued into the 21 st century, with outbreaks in India, Iran, Vietnam, and several African countries [9]. Some recent outbreaks occurred in Haiti and Nigeria between 2010 and 2011 and South Sudan, Tanzania, Iraq, Kenya and Cuba from 2015 to 2016 [8]. Also between 2017 and 2018, about 1,084,191 suspected cholera cases with 2,267 associated deaths were reported in war-torn Yemen by the WHO [10]. Although cholera claim lives easily, it can be eradicated by effective control measures.

John Snow is recognized as one of the earliest researchers in cholera transmission owing to his work on the Soho Cholera outbreak in 1854 [11]. He performed his pioneered work on cholera in London where he established an association between cholera transmission and contaminated water. He discovered that the method of prevention and control of cholera was to trace its source back to the drinking water. He realized that by closing the broad street pump that supplied contaminated water to other areas, the transfer of the cholera bacteria could be eradicated from the area. This discovery paved way for further studies on cholera and later in 1883, Robert Koch described the causative agent for cholera as a curved bacillus and named it Vibrio cholerae [3]. Before the late 1970, it was believed that person-to-person transmission of cholera is the main mode of transmission though there are other means of transmission. The faeces or vomit of a contaminated person carries the Vibrio cholerae bacteria, and it spreads through the population by the faecal-oral route. Cholera is frequently transmitted by water sources contaminated with Vibrio cholerae, although contaminated foods such as raw shellfish and oyster may also transmit the bacteria [12]. When contaminated food is ingested into the body, the bacteria can survive passing through the acidic conditions of the stomach. Inside the small intestine where absorption takes place, the Vibrio cholerae bacteria attaches itself to the intestinal wall and starts producing cholera toxin. The toxin enters the intestinal cells causing them to release water and ions, including sodium and chloride ions [13]. The saltwater environment created inside the intestine can by osmosis draw up to a further six litres of water into the intestine each day. This results in the production of watery diarrhea and severe dehydration. The symptoms of cholera are the direct results of this attack on the body which includes the intense diarrhea and vomiting which results in general body weakness and dizziness.
It is possible to eradicate cholera with appropriate measures such as safe drinking water and noncontaminated foods. Measures for the prevention of cholera include, but not limited to, provision of treated water and proper sanitation to cholera-endemic communities. Also, some protection can be obtained from oral vaccines for immunity. This oral vaccine is about $80 \%$ effective for a period of three months after a single oral dose is administered to adults who are between the ages of 18 and 64 years.

Based on this knowledge, effective prevention and intervention strategies have been developed over the years through the formulation of mathematical models to provide a unique approach to gain basic insight into cholera dynamics [14]. For example [15], a cholera model is built that explicitly incorporated the environment as a component in a regular $S I R$ system to form a combined environment to the human $(S I R B)$ epidemiological model. This model enabled a careful study on the complex interaction between the human host and environmental pathogen. This has led towards a better understanding of the cholera transmission mechanism and has also motivated the development of several other cholera models.

A more general model which took into account the different infective states of Vibrio cholerae was developed in [16]. Their model consisted of five equations which described the dynamics of a susceptible, infectious, and removed human population. It also incorporated the dynamics of a hyperinfective state and lower-infective states of Vibrio cholerae population. In their model, they assumed the total population $N$ to be constant and also assumed a constant birth and death rate $b$. Infection is caused by ingestion of contaminated water with either $\mathrm{BH}$ (hyperinfective vibrios) or BL (lower-infective vibrios).

Building on the work in [15], Mukandavire et al. [17] developed a model by incorporating a human-to-human transmission factor. That is, the interactions between the susceptible and infective class. They were able to estimate the basic reproduction number $\left(R_{0}\right)$ for the 2008-2009 cholera outbreak in Zimbabwe. They again presented a model fitted to the Zimbabwean data that provided insights into the nature of the epidemic in Zimbabwe. This helped, on a larger scale, to control cholera at a global level. Specifically, they used the Zimbabwean data to derive estimates of the basic reproductive number $\left(R_{0}\right)$ of cholera on a regional basis.

In another development, Tuite et al. [18] built an SIR compartmental transmission model that characterized the population as susceptible to infection, infected, and infectious to others. They also characterized the recovered or otherwise were removed from risk to further infection. They assumed that cholera could be transmitted through either contaminated water or by being in close contact with an infected person. They also found out that waterborne transmission was a critical mode of transmission. They therefore added a water compartment to the model. As a control measure, they introduced vaccination into the model by moving persons from the susceptible compartment to recovered or immune compartment when vaccination was completed. They also explored the relative effect of replacing 
vaccination as a control with provision of clean water which reduced the number of persons who are susceptible to infection through contaminated water. With the advance in sanitation systems and availability of portable water, together with massive awareness of personal hygiene, cholera incidence was no longer a problem.

Furthermore, Sun et al. [19] proposed a mathematical model that described the transmission of cholera in China. In their model, they computed the basic reproduction number and the global dynamics of the system. Although they considered control parameters which were immunization coverage rate and environmental management especially drinking water, they failed to apply optimal control analysis. Hence, they were not able to use Pontryagin's maximum principle, which is one of the methods used in control theory problems, to find the best possible control for taking a dynamical system from one state to another. They also did not consider features of the bacteria such as the carrying capacity of the bacteria in the environment in which they evolve. Therefore, they could not get the controls to work effectively.

Ainea et al. [20] also proposed and analyzed a deterministic cholera model in Tanzania to help the eradication of cholera. Necessary conditions of the optimal control problem were analyzed by applying Pontryagin's maximum principle. They incorporated two control strategies which were human educational campaign and treatment of water. Nevertheless, they did not analytically calculate their model to determine the basic reproduction number which serves as a threshold value for the dynamics of the system and the disease.

Again, Ezeagu et al. [21] also considered a mathematical model for the transmission of cholera dynamics with a class of quarantined and vaccination parameter as control strategies. They determined the constant solutions to the model, that is, the disease-free and endemic equilibrium points. They also used the next-generation matrix to determine the basic reproduction number around the diseasefree equilibrium points. Their result showed that by applying the controls the disease can be eradicated. Moreover, many of such works have been studied and recorded in $[2,22-26]$.

The motivation for this paper comes from the various studies carried out in the transmission dynamics of cholera. However, in this paper, we took into account a saturated incidence rate where the number of effective contacts between infected and susceptible individuals may saturate at high infected levels due to crowding of infected individuals or due to the application of some measures by the susceptible individuals for the spread of the disease. This has been observed to play an important role on the dynamics of the transmission of diseases. Furthermore, by not just considering the bacteria population as a compartment, we assume a logistic equation term for the growth of the bacteria in the environment. This is incorporated to help ascertain the behaviour of the bacteria within a confined environment, thus, making it possible to track the survival rate of the bacteria since the bacteria population gets smaller and smaller as the population size approaches a maximum. The carrying capacity further acts as a control measure since it becomes a limiting value of the bacteria population. Finally, some control measures were also applied to ascertain the transmission dynamics of cholera.

\section{Model Formulation}

In this section, we develop a model for cholera transmission within a well-mixed population. It is a combined system of the human population $(S I R)$ and the bacteria $(B)$ in the environment as a compartment to form an $S I R B$ model. The total human population at time $t$ is denoted by $N(t)$, and it is divided among three different compartments. These are the susceptible individuals denoted by $(S)$, infectious individuals denoted by $(I)$, and the recovered individuals denoted by $(R)$.

In order to formulate the model mathematically, we assume that individuals are recruited into the susceptible compartment at a rate $\Delta$. A proportion $\tau$ of the susceptible individuals are vaccinated which offers some immunity against the infection. As indicated in [27], we further assume that individuals that recover from cholera as a result of receiving vaccination develop an immunity that protect them from being susceptible to the disease for several years. However, susceptible individuals can be infected either by coming into contact with an infected person or the bacteria in the environment. Infected individuals transmit cholera to susceptible individuals at a rate $\beta_{h}$ and susceptible individuals contract the bacteria from the environment at a rate $\beta_{e}$. The parameter $\omega$ is the concentration of vibrios. The force of infection is thus represented by $\Upsilon_{h}=\left(\beta_{e} S B /(B+\omega)\right)$ $+\left(\beta_{h} S I /(1+\alpha I)\right)$, where $I /(1+\alpha I)$ is the saturation incidence rate with constant $\alpha$ adjusting the appropriate form of the incidence which determines the rate of new infection. As a result, if $\alpha=0$, then the corresponding incidence reduces to the standard bilinear based on the mass action law. If $\alpha>0$, then the corresponding incidence represents a consequence of saturation effects: when the infected number is high, the incidence rate will respond more slowly than linearly to the increase in $I$.

Since a confined area is being considered, that is, the environment, the growth of the bacteria is represented by using a logistic equation $\lambda_{B}=r B(1-(B / K))$, where $r$ is the growth rate and $K$ is the carrying capacity of the bacteria. Control measures $c_{1}$ which is educating susceptible individuals and $c_{2}$ which is the treatment of water bodies are also considered. Hence, susceptible individuals contract the cholera disease from an infected individual at a reduced rate $\left(1-c_{1}\right) \Upsilon_{h}$, where $c_{1} \in(0,1)$ measures the effectiveness of the educational campaign. If $c_{1}=0$, then the educational campaign will be useless, but if $c_{1}=1$, then the educational campaign is $100 \%$ effective in preventing cholera. Natural death rate $\mu$ and a disease-induced death rate $\gamma$ are considered. Also, there is a decay rate $\delta$ for the bacteria population. The general transmission dynamics is shown in Figure 1.

Table 1 briefly describes the parameters as being used in the compartmental model. 


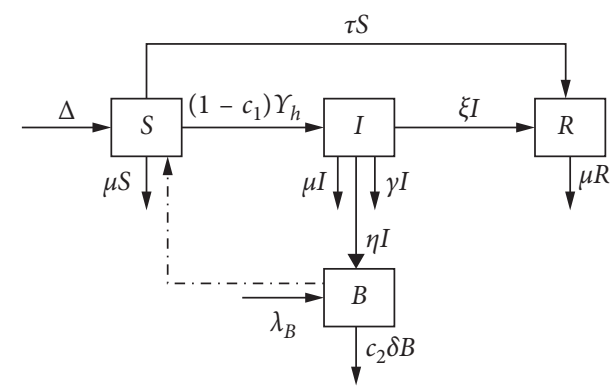

FIgURE 1: The transmission dynamics of cholera.

TABle 1: Description of parameters involved in the flow chart diagram.

\begin{tabular}{lc}
\hline Parameter & Description \\
\hline$\beta_{e}$ & Rate of Vibrio ingestion in the environment \\
$\beta_{h}$ & Human-to-human interaction \\
$c_{1}$ & Educating individuals on reducing the contact \\
$c_{2}$ & between infected people \\
$\omega$ & Treating water bodies in the environment \\
$K$ & Concentration of Vibrio cholerae in the environment \\
$r$ & Carrying capacity of Vibrio cholerae \\
$\Delta$ & Growth rate of each Vibrio cholerae in the \\
$\Xi$ & environment \\
$\eta$ & Recruitment rate \\
$\delta$ & Recovery rate \\
$\mu$ & Rate of human contribution to Vibrio cholerae \\
$\gamma$ & Decay rate of vibrios \\
$\tau$ & Natural death rate \\
$\alpha$ & Disease-induced death rate \\
\hline
\end{tabular}

\section{Model Analysis}

From Figure 1, we can express the time evolution of the population states in the following deterministic nonlinear ordinary differential equations:

$$
\left\{\begin{array}{l}
\frac{\mathrm{d} S}{\mathrm{~d} t}=\Delta-\left(1-c_{1}\right) \Upsilon_{h}-\mu S-\tau S, \\
\frac{\mathrm{d} I}{\mathrm{~d} t}=\left(1-c_{1}\right) \Upsilon_{h}-(\mu+\gamma+\xi+\eta) I, \\
\frac{\mathrm{d} R}{\mathrm{~d} t}=\xi I+\tau S-\mu R, \\
\frac{\mathrm{d} B}{\mathrm{~d} t}=\eta I+\lambda_{B}-c_{2} \delta B
\end{array}\right.
$$

\subsection{Basic Properties of the Model}

Theorem 1. The biologically feasible region $\Omega$ is given by the following set:

$$
\Omega=\left\{(S, I, R, B) \in R_{+}^{4}: S+I+R ; \quad N \leq \frac{\Delta}{\mu}\right\}
$$

with nonnegative initial conditions $S(0)>0$ and $I(0), R(0)$, and $B(0) \geq 0$. It is bounded, positively invariant, and attracting with respect to system (1) for all $t>0$.

Proof. We sum up system (1) that makes up $\mathrm{d} N / \mathrm{d} t$ to obtain

$$
\begin{aligned}
\frac{\mathrm{d} N}{\mathrm{~d} t} & =\Delta-\mu N, \\
\frac{\mathrm{d} N}{\mathrm{~d} t}+\mu N & =\Delta .
\end{aligned}
$$

The solution of equation (3) is given by

$$
N(t)=\frac{\Delta}{\mu}+C e^{-\mu t} .
$$

Considering the initial conditions at $t=0$, let $N(0)$ be the initial value of the total human population. Therefore,

$$
\begin{aligned}
N(0) & =\frac{\Delta}{\mu}+C e^{-\mu(0)}, \\
N(0) & =\frac{\Delta}{\mu}+C, \\
C & =N(0)-\frac{\Delta}{\mu} .
\end{aligned}
$$

Hence,

$$
N(t)=\frac{\Delta}{\mu}+\left(N(0)-\frac{\Delta}{\mu}\right) e^{-\mu t} .
$$

As $N(t) \longrightarrow \Delta / \mu$, every solution $N(t)$ of equation (3) satisfies the following condition:

$$
0 \leq N(t) \leq N(0) e^{-\mu t}+\frac{\Delta}{\mu}\left(1-e^{-\mu t}\right),
$$

where $N(0)$ is the sum of all the initial conditions of the variables of system (1). The total human population at time $t$ would be bounded above by $\Delta / \mu$ if $N(0) \leq \Delta / \mu$. Hence, for any solution of system (1), it either remains in or approaches $\Omega$ asymptotically. Thus, $\Omega$ is positively invariant and attracting with respect to the model.

3.2. Positivity and Boundedness of Solutions. Since the model monitors human population, we show that all the state variables remain nonnegative for all times.

Theorem 2. Let $\Omega=\left\{S, I, R, B \in \mathbb{R}_{+}^{4}: S(0)>0, I(0)>0\right.$, $R(0)>0, B(0)>0\}$, then the solutions of $S(t), I(t), R(t)$, and $B(t)$ of system (1) are positive for all $t \geq 0$.

Proof. Considering

$$
\widehat{t}=\sup \{t>0: S(t)>0 \text { and } I(t), R(t), B(t) \geq 0\} .
$$

This implies that

$$
S(t)>0 \text { and } I(t), R(t), B(t) \geq 0, \quad \forall t \in[0, t) .
$$


Considering the first equation in system (1),

$$
\begin{aligned}
\frac{\mathrm{d} S}{\mathrm{~d} t} & =\Delta-\left(1-c_{1}\right) \Upsilon_{h}-\mu S-\tau S \\
& =\Delta-\left(1-c_{1}\right) \Upsilon_{h}-(\mu+r) S .
\end{aligned}
$$

From equation (10), since $\Delta$ is positive, we obtain

$$
\frac{\mathrm{d} S}{\mathrm{~d} t} \geq-\left(1-c_{1}\right) \Upsilon_{h}-(\mu+r) S, \quad \forall t \in[0, \widehat{t}) .
$$

Separation of variables and then integrating both sides gives

$$
\begin{gathered}
\frac{\mathrm{d} S}{S} \geq-\left[\left(1-c_{1}\right) \Upsilon_{h}+(\mu+r)\right] \mathrm{d} t, \\
\ln \left(\frac{S(\widehat{t})}{S(0)}\right) \geq\left[-\int_{0}^{\widehat{t}}\left(1-c_{1}\right) \Upsilon_{h} \mathrm{~d} t-\mu \widehat{t}-r \widehat{t}\right], \\
S(\widehat{t}) \geq S(0) \exp \left[-\int_{0}^{\widehat{t}}\left(1-c_{1}\right) \Upsilon_{h} \mathrm{~d} t-\mu \widehat{t}-r \widehat{t}\right]>0 .
\end{gathered}
$$

In a similar manner, the solution for the rest of the state variables is obtained as follows:

$$
\begin{aligned}
& I(\widehat{t}) \geq I(0) \exp [-\mu \widehat{t}-\gamma \widehat{t}-\xi \widehat{t}-\eta \widehat{t}] \geq 0, \\
& R(\widehat{t}) \geq R(0) \exp [-\mu t] \geq 0, \\
& B(\widehat{t}) \geq B(0) \exp -\left(c_{2} \delta B\right) \widehat{t} \geq 0 .
\end{aligned}
$$

Therefore, $S(t)>0$ and $I(t), R(t)$, and $B(t) \geq 0$ for all $t>0$, given the initial conditions.

3.3. Model Steady States. In this section, the equilibrium points of system (1), that is, the disease-free equilibrium point (DFE) denoted by $\mathscr{E}_{0}$ and endemic equilibrium point (EE) denoted by $\mathscr{E}^{*}$, are determined. The stability of the model is investigated using the basic reproduction number, $R_{0}$.

3.3.1. Disease-Free Equilibrium Point (DFE), $\mathscr{E}_{0}$. In the absence of Vibrio cholerae and by resolving system (1), the DFE state is obtained as

$$
\mathscr{E}_{0}=\left(S_{0}, I_{0}, R_{0}, B_{0}\right)=\left(\frac{\Delta}{\mu+\tau}, 0,0,0\right) .
$$

3.3.2. Endemic Equilibrium Point (EE), $\mathscr{E}^{*}$. The endemic equilibrium point, $\mathscr{E}^{*}=\left(S^{*}, I^{*}, R^{*}, B^{*}\right)$, is the nontrivial equilibrium point of system (1). By resolving these equations, we can correspondingly acquire the equilibrium solutions of different states as

$$
\left\{\begin{array}{l}
S^{*}\left(B^{*}\right)=\frac{\Delta-(\mu+\gamma+\xi+\eta)\left(c_{2} \delta B^{*}-r B^{*}\left(1-\left(B^{*} / K\right)\right)\right)}{(\mu+\tau) \eta}, \\
I^{*}\left(B^{*}\right)=\frac{c_{2} \delta B^{*}-r B^{*}\left(1-\left(B^{*} / K\right)\right)}{\eta}, \\
R^{*}\left(B^{*}\right)=\frac{\xi\left(c_{2} \delta B^{*}-r B^{*}\left(1-\left(B^{*} / K\right)\right)\right)+\tau\left(\Delta-(\mu+\gamma+\xi+\eta)\left(c_{2} \delta B^{*}-r B^{*}\left(1+\left(B^{*} / K\right)\right)\right)\right)}{\eta^{2} \mu(\mu+\tau)}, \\
B^{*}=\left(K-\frac{K \delta c_{2}}{r} \pm\left(\frac{\sqrt{\left(M_{1} M_{2}+M_{3}\right)}}{2\left(r(\gamma+\eta+\mu+\xi)(-\alpha(\mu+\tau))+\eta^{3}\left(-1+c_{1}\right) \beta_{h}\right)}\right)\right)
\end{array}\right.
$$

where

$$
\begin{aligned}
& M_{1}=K(\gamma+\mu+\xi+\eta)\left(\alpha(\mu+\tau)-\eta^{3}\left(-1+c_{1}\right) \beta_{h}\right) \\
& M_{2}=\left(-4 r \eta\left((\gamma+\eta+\mu+\xi)(\mu+\tau)+\Delta \eta^{2}\left(-1+c_{1}\right) \beta_{h}\right)\right) \\
& M_{3}=K(\gamma+\mu+\xi+\eta)\left(r-\delta c_{2}\right)^{2}\left(\alpha(\mu+\tau)-\eta^{3}\left(-1+c_{1}\right) \beta_{h}\right) .
\end{aligned}
$$

3.3.3. Basic Reproduction Number, $R_{0}$. The basic reproduction number is the expected number of secondary cases that occur when one Vibrio cholerae bacterium is introduced into a wholly susceptible environment as well as one infectious individual is also being introduced into a completely susceptible population [28]. It is calculated using the method 
in [29], and it is obtained as

$$
R_{0}^{c}=\frac{\beta_{h} \Delta\left(1-c_{1}\right)}{(\mu+\gamma+\xi+\eta)(\mu+\tau)}+\frac{\beta_{e} \Delta \eta}{\omega(\mu+\gamma+\xi+\eta)\left(r+c_{2} \delta\right)(\mu+\tau)} .
$$

The superscript $c$ is used to emphasize the model with controls compared to the basic reproduction number when the control parameters are taken out of the model which is given as

$$
R_{0}=\frac{\beta_{h} \Delta}{(\mu+\gamma+\xi+\eta)(\mu+\tau)}+\frac{\beta_{e} \Delta \eta}{\omega r(\mu+\gamma+\xi+\eta)(\mu+\tau)} .
$$

It can be seen that $R_{0}^{c}<R_{0}$ which shows that both $R_{0}^{c}$ and $R_{0}$ describe how humans transfer cholera to other humans $\left(\beta_{h}\right)$ and also how the bacteria in the environment contribute to the transmission of cholera $\left(\beta_{e}\right)$. It further shows how each of the controls can reduce the value of $R_{0}^{c}$ below 1 so that the disease will be eradicated.

3.3.4. Analysis of $R_{0}$ with Control Measures. The basic reproduction number in equation (17) is again used to show the effectiveness of the respective control measures when applied. If educating individuals $c_{1}$ on reducing the contact between infected person and susceptible individuals, as well as infected individuals understanding the mode of transmission and prevention of cholera, is the only control measure being applied, that is, $c_{1} \neq 0$ and $c_{2}=0$, then the basic reproduction number is given by

$$
R_{0}^{c_{1}}=\frac{\beta_{h} \Delta\left(1-c_{1}\right)}{(\mu+\gamma+\xi+\eta)(\mu+\tau)}+\frac{\beta_{e} \Delta \eta}{\omega r(\mu+\gamma+\xi+\eta)(\mu+\tau)} .
$$

If treatment of water bodies in the environment $c_{2}$ is also the only control measure being applied, then the basic reproduction number is obtained as

$$
R_{0}^{c_{2}}=\frac{\beta_{h} \Delta}{(\mu+\gamma+\xi+\eta)(\mu+\tau)}+\frac{\beta_{e} \Delta \eta}{\omega(\mu+\gamma+\xi+\eta)\left(r+c_{2} \delta\right)(\mu+\tau)} .
$$

3.4. Local Stability of DFE. The local stability of the DFE is obtained by linearizing system (1), that is, by computing its Jacobian matrix, $J\left(\mathscr{E}_{0}\right)$. The Jacobian matrix is computed by differentiating each equation in system (1) with respect to the state variables $S, I, R$, and $B$.

Theorem 3. If $R_{0} \leq 1$, then the disease-free equilibrium state which is given by $\mathscr{E}_{0}$ is locally asymptotically stable in the region defined by $\Omega=\left\{(S, I, R, B) \in R_{+}^{4}: S+I+R ; N \leq \Delta / \mu\right\}$ and unstable when $R_{0}>1$.

Proof. The disease-free equilibrium state is locally stable if all eigenvalues of the Jacobian matrix $J\left(\mathscr{E}_{0}\right)$ are negative. The matrix has all eigenvalues negative only if the trace of $J\left(\mathscr{E}_{0}\right)<0$ and determinant of $J\left(\mathscr{E}_{0}\right)>0$. We then linearize system (1) around $\mathscr{E}_{0}$, to obtain

$$
J\left(\mathscr{E}_{0}\right)=\left(\begin{array}{cccc}
-(\mu+\tau) & -\left(1-c_{1}\right)\left(\frac{\beta_{h} \Delta}{\mu+\tau}\right) & 0 & -\left(1-c_{1}\right)\left(\frac{\beta_{e} \Delta}{(\mu+\tau) \omega}\right) \\
0 & \left(1-c_{1}\right)\left(\frac{\beta_{h} \Delta}{\mu+\tau}\right)-(\mu+\gamma+\xi+\eta) & 0 & \left(1-c_{1}\right)\left(\frac{\beta_{e} \Delta}{(\mu+\tau) \omega}\right) \\
\tau & \xi & -\mu & 0 \\
0 & \eta & 0 & r-c_{2} \delta
\end{array}\right) .
$$

The matrix $J\left(\mathscr{E}_{0}\right)$ has eigenvalues $-(\mu+\tau)$ and $-\mu$ having negative real parts. This implies that $J\left(\mathscr{E}_{0}\right)$ can be reduced to obtain

$$
z=\left|\begin{array}{cc}
\left(\left(1-c_{1}\right)\left(\frac{\beta_{h} \Delta}{\mu+\tau}\right)-(\mu+\gamma+\xi+\eta)\right)-\lambda & \left(1-c_{1}\right)\left(\frac{\beta_{e} \Delta}{(\mu+\tau) \omega}\right) \\
\eta & \left(r-c_{2} \delta\right)-\lambda
\end{array}\right|=0 .
$$


From equation (22), the characteristic polynomial can be written as

$$
\lambda^{2}+b_{1} \lambda+b_{2}=0
$$

where

$$
\begin{aligned}
b_{1}= & \left(\left(-r+c_{2} \delta\right)-\left(\left(1-c_{1}\right)\left(\frac{\beta_{h} \Delta}{\mu+\tau}\right)\right)+(\mu+\gamma+\xi+\eta)\right), \\
b_{2}= & \left(r-c_{2} \delta\right)\left(\left(1-c_{1}\right)\left(\frac{\beta_{h} \Delta}{\mu+\tau}\right)-(\mu+\gamma+\xi+\eta)\right) \\
& -\eta\left(1-c_{1}\right)\left(\frac{\beta_{e} \Delta}{(\mu+\tau) \omega}\right) .
\end{aligned}
$$

By applying the Routh-Hurwitz criteria for a $2 \times 2$ matrix in equation (22), it requires that $b_{1}$ and $b_{2}$ in equation (23) be greater than zero which is a necessary condition for the root of the polynomial to be negative or have a negative real part. Thus, we have

$$
\begin{aligned}
b_{1}= & r+\delta c_{2}+\frac{\beta_{e} \Delta \eta}{\omega\left(r+c_{2} \delta\right)(\mu+\tau)}+(\mu+\gamma+\xi+\eta)\left(1-R_{0}\right), \\
b_{2}= & \frac{\Delta \beta_{e} \eta \omega\left(1-c_{1}\right)}{(\mu+\tau)\left(r-\delta c_{2}\right)}+\frac{\beta_{e} \Delta \eta}{\omega\left(r+c_{2} \delta\right)(\mu+\tau)} \\
& +(\mu+\eta+\gamma+\xi)\left(1-R_{0}\right) .
\end{aligned}
$$

We observe that $b_{1}>0$ and $b_{2}>0$ if $R_{0} \leq 1$ which implies that the disease-free equilibrium is locally stable.

3.5. Global Stability of DFE. In this section, the disease-free equilibrium state is shown to be globally stable by using Lyapunov's theorem.

Theorem 4. The disease-free equilibrium is globally asymptotically stable if $R_{0}<1$ and unstable otherwise.

Proof. Applying the method in [30], let $V(I, B)$ be a Lyapunov function with some nonnegative parameters, $\theta$ and $\theta_{1}$, such that

$$
V(I, B)=\theta I+\theta_{1} B .
$$

Differentiating the Lyapunov function with respect to time gives

$$
\frac{\mathrm{d} V}{\mathrm{~d} t}=\theta \frac{\mathrm{d} I}{\mathrm{~d} t}+\theta_{1} \frac{\mathrm{d} B}{\mathrm{~d} t} .
$$

Substituting $\mathrm{d} I / \mathrm{d} t$ and $\mathrm{d} B / \mathrm{d} t$ into equation (27) gives

$$
\begin{aligned}
\frac{\mathrm{d} V}{\mathrm{~d} t} \leq & \theta\left[\left(1-c_{1}\right) \Upsilon_{h}-(\mu+\gamma+\xi+\eta) I\right]+\theta_{1}\left[\eta I+\lambda_{B}-c_{2} \delta B\right] \\
\leq & \theta\left[\left(1-c_{1}\right)\left(\frac{\beta_{e} S B}{B+\omega}+\frac{\beta_{h} S I}{1+\alpha I}\right)-(\mu+\gamma+\xi+\eta)\right] \\
& +\theta_{1}\left[\eta I+r B\left(1-\frac{B}{K}\right)-c_{2} \delta B\right] \\
\leq & \left\{\theta\left(1-c_{1}\right)\left(\frac{\beta_{e} S B}{B+\omega}\right)+\theta\left(1-c_{1}\right)\left(\frac{\beta_{h} S I}{1+\alpha I}\right)\right. \\
& -\theta(\mu+\gamma+\xi+\eta) I+\theta_{1} \eta I+\theta_{1} r B\left(1-\frac{B}{K}\right)-\theta_{1} c_{2} \delta B .
\end{aligned}
$$

By grouping like terms for $I$ and $B$, we have

$$
\begin{aligned}
\frac{\mathrm{d} V}{\mathrm{~d} t} \leq & \left\{\theta\left(1-c_{1}\right)\left(\frac{\beta_{h} S I}{1+\alpha I}\right)+\theta_{1} \eta I-\theta(\mu+\gamma+\xi+\eta) I\right. \\
& +\theta\left(1-c_{1}\right)\left(\frac{\beta_{e} S B}{B+\omega}\right)+\theta_{1} r B\left(1-\frac{B}{K}\right)-\theta_{1} c_{2} \delta B \\
\leq & \left\{\theta\left(1-c_{1}\right)\left(\frac{\beta_{h} S I}{1+\alpha I}\right)+I\left(\theta_{1} \eta-\theta(\mu+\gamma+\xi+\eta)\right)\right. \\
& +\theta\left(1-c_{1}\right)\left(\frac{\beta_{e} S B}{B+\omega}\right)+B\left(\theta_{1}\left(r-\frac{r B}{K}\right)-\theta_{1} c_{2} \delta\right) .
\end{aligned}
$$

After forming the Lyapunov function given as $V$ on the space of the two state variables $(I, B)$, it is observed that if $I(t)$ and $B(t)$ at $\mathscr{E}_{0}$ are globally stable (that is, $I=0$ and $B=0)$, then $S(t) \longrightarrow \Delta /(\mu+\tau)$. Hence, substituting the values of $I=0$ and $B=0$ gives

$$
\frac{\mathrm{d} V}{\mathrm{~d} t}=\left(\theta_{1} \eta-\theta(\mu+\gamma+\xi+\eta)\right) I-\left(\theta_{1} c_{2} \delta\right) B .
$$

Equating the coefficients of $I$ in equation (30) to zero which correspond to the disease-free equilibrium and applying the multiplication property of equality which states that if $a=b$ and $c=d$, then $a c=b d$. We can obtain equation (31) from equation (30), where equation (31) is given by

$$
\theta_{1} \eta=\theta(\mu+\gamma+\xi+\eta)
$$

Choosing $\theta_{1}=(\mu+\gamma+\xi+\eta)$ and $\theta=\eta$ and then substituting into equation (30) give

$$
\frac{\mathrm{d} V}{\mathrm{~d} t}=[\eta(\mu+\gamma+\xi+\eta)-\eta(\mu+\gamma+\xi+\eta)] I-\left[(\mu+\gamma+\xi+\eta) c_{2} \delta\right] B .
$$

After some algebraic calculations, we obtain 


$$
\begin{aligned}
\frac{\mathrm{d} V}{\mathrm{~d} t}= & {\left[\left(\frac{\beta_{h} \delta\left(1-c_{1}\right)}{\mu+\tau}+\frac{\beta_{e} \delta \eta}{\omega\left(r+c_{2} \delta\right)(\mu+\tau)}\right) c_{2} \delta\right.} \\
& \left.\cdot(\mu+\gamma+\xi+\eta)\left[R_{0}-1\right]\right] B .
\end{aligned}
$$

Therefore, $\mathrm{d} V / \mathrm{d} t=0$ if and only if $B=0$ and $\mathrm{d} V / \mathrm{d} t<0$ if $R_{0}<1$. As a result, the largest compact invariant set in $\{(S, I, R, B) \in \Omega: \mathrm{d} V / \mathrm{d} t=0\}$ is the singleton set $\left\{\mathscr{E}_{0}\right\}$. From La Salle's invariance principle, it can be concluded that $\left\{\mathscr{E}_{0}\right\}$ is globally asymptotically stable in $\Omega$ if $R_{0}<1$.

\subsection{Local Stability of EE}

Theorem 5. The endemic equilibrium point which is defined by $\mathscr{E}^{*}$ is locally asymptotically stable in the region defined by $\Omega=\left\{(S, I, R, B) \in R_{+}^{4}: S+I+R ; N \leq \Delta / \mu\right\}$ if $R_{0}>1$, and it becomes unstable if $R_{0} \leq 1$.

Proof. Based on the approach by $[31,32]$, the theorem is proved from the EE defined in equation (15). Since all the values are positive, the value of $B^{*}$ is expressed in terms of $R_{0}$ which gives

$$
\begin{aligned}
B^{*}= & \left(\frac{K r-K \delta c_{2}}{r}\right)^{2}+4 \eta\left(K(\mu+\gamma+\xi+\eta) \alpha(\mu+\tau)+\eta^{3}\left(r-\delta c_{2}\right)^{2}\left(1-c_{1}\right) \beta_{h}\right)\left(-(\mu+\gamma+\xi+\eta)(\mu+\tau)\left(r-\delta c_{2}\right)^{2}\right. \\
& \left.+(\mu+\tau)(\mu+\gamma+\xi+\eta)\left(r-\delta c_{2}\right)^{2} R_{0}+\frac{\beta_{e} \Delta \eta\left(r-\delta c_{2}\right)}{\omega}\right) \\
= & \left(\frac{K r-K \delta c_{2}}{r}\right)^{2}+4 \eta\left(K(\mu+\gamma+\xi+\eta) \alpha(\mu+\tau)+\eta^{3}\left(r-\delta c_{2}\right)^{2}\left(1-c_{1}\right) \beta_{h}\right)\left(R_{0}-1\right) \\
& {\left[(\mu+\tau)(\mu+\gamma+\xi+\eta)\left(r-\delta c_{2}\right)^{2}\right]+\frac{\beta_{e} \Delta \eta\left(r-\delta c_{2}\right)}{\omega} . }
\end{aligned}
$$

Since $B^{*}$ is positive when $R_{0}>1$ implies that the EE is locally asymptotically stable.

3.7. Global Stability of EE. Considering the method used in [19], a Lyapunov function $V$ is defined as

$$
\begin{aligned}
V= & \int_{S^{*}}^{S}\left(1-\frac{S^{*}}{X}\right) \mathrm{d} X+\int_{I^{*}}^{I}\left(1-\frac{I^{*}}{X}\right) \mathrm{d} X \\
& +\frac{\left(1-c_{1}\right)}{\eta I^{*}}\left(\frac{\beta_{e} S^{*} B^{*}}{B^{*}+\omega}+\frac{\beta_{h} S^{*} I^{*}}{1+\alpha I^{*}}\right) \int_{B^{*}}^{B}\left(1-\frac{B^{*}}{X}\right) \mathrm{d} X .
\end{aligned}
$$

The derivative of $V$ is found along solutions of system (1) to obtain

$$
\begin{aligned}
\dot{V}= & \left(1-\frac{S^{*}}{S}\right) \dot{S}+\left(1-\frac{I^{*}}{I}\right) \dot{I} \\
& +\frac{\left(1-c_{1}\right)}{\eta I^{*}}\left(\frac{\beta_{e} S^{*} B^{*}}{B^{*}+\omega}+\frac{\beta_{h} S^{*} I^{*}}{1+\alpha I^{*}}\right)\left(1-\frac{\dot{B}}{B}\right) \dot{B} .
\end{aligned}
$$

After some algebraic calculations yielded,

$$
\begin{aligned}
\left(1-\frac{S^{*}}{S}\right) \dot{S} & =\left\{\left(\left(1-c_{1}\right)\left(\frac{\beta_{e} S^{*} B^{*}}{B^{*}+\omega}+\frac{\beta_{h} S^{*} I^{*}}{1+\alpha I^{*}}\right)\right)-\left(1-c_{1}\right)\left(\left(1-c_{1}\right)\left(\frac{\beta_{e} S^{*} B^{*}}{B^{*}+\omega}+\frac{\beta_{h} S^{*} I^{*}}{1+\alpha I^{*}}\right)\right)+\left(1-\frac{S^{*}}{S}\right)\left(\mu S^{*}-\mu S\right)+\left(1-\frac{S^{*}}{S}\right)\left(\tau S^{*}-\tau S\right)\right. \\
& \leq\left(1-c_{1}\right)\left(\frac{\beta_{e} S^{*} B^{*}}{B^{*}+\omega}+\frac{\beta_{h} S^{*} I^{*}}{1+\alpha I^{*}}\right)\left(1-\frac{S^{*}}{S}\right)+\tau S^{*}\left(1-\frac{S^{*}}{S}\right)[1-\tau S], \\
\left(1-\frac{I^{*}}{I}\right) \dot{I} & =\left(1-\frac{I^{*}}{I}\right)\left(\left(1-c_{1}\right)\left(\frac{\beta_{e} S^{*} B^{*}}{B^{*}+\omega}+\frac{\beta_{h} S^{*} I^{*}}{1+\alpha I^{*}}\right)-\left(1-c_{1}\right)\left(\frac{\beta_{e} S^{*} B^{*}}{B^{*}+\omega}+\frac{\beta_{h} S^{*} I^{*}}{1+\alpha I^{*}}\right) \frac{I}{I^{*}}\right) \\
& =\left\{\left(1-c_{1}\right)\left(\frac{\beta_{e} S^{*} B^{*}}{B^{*}+\omega}+\frac{\beta_{h} S^{*} I^{*}}{1+\alpha I^{*}}\right)\left(1-\frac{I^{*}}{I}\right)\left(\frac{S\left(\beta_{e} S B /(B+\omega)\right)}{S^{*}\left(\beta_{e} S^{*} B^{*} /\left(B^{*}+\omega\right)\right)}-\frac{I}{I^{*}}\right)+\left(1-c_{1}\right)\left(\frac{\beta_{h} S^{*} I^{*}}{1+\alpha I^{*}}\right)\left(1-\frac{I^{*}}{I}\right)\left(\frac{S I}{S^{*} I^{*}}-\frac{I}{I^{*}}\right),\right. \\
& \frac{\left(1-c_{1}\right)}{\eta I^{*}}\left(\frac{\beta_{e} S^{*} B^{*}}{B^{*}+\omega}\right)\left(1-\frac{B^{*}}{B}\right) \dot{B}=\left(1-c_{1}\right)\left(\frac{\beta_{e} S^{*} B^{*}}{B^{*}+\omega}\right)\left(1-\frac{B^{*}}{B}\right)\left(\frac{I}{I^{*}}-\frac{B}{B^{*}}\right) .
\end{aligned}
$$


Therefore,

$$
\dot{V} \leq\left\{\begin{array}{l}
\left(1-c_{1}\right) \beta_{e} S^{*} \frac{B^{*}}{B^{*}+\omega}\left(1-\frac{S^{*}}{S}\right)\left[1-\frac{S B\left(B^{*}+\omega\right)}{S^{*} B^{*}\left(B^{*}+\omega\right)}\right]+\left(1-c_{1}\right)\left(\frac{\beta_{h} S^{*} I^{*}}{1+\alpha I}\right)\left(1-\frac{S^{*}}{S}\right) \\
+(1-S I)+\left(1-c_{1}\right) \beta_{e} S^{*} \frac{B^{*}}{B^{*}+\omega}\left(1-\frac{I *}{I}\right)\left(\frac{S^{*}}{S}\right)\left[\frac{S B\left(B^{*}+\omega\right)}{S^{*} B^{*}\left(B^{*}+\omega\right)}-\frac{I}{I^{*}}\right) \\
+\left(1-c_{1}\right)\left(\frac{\beta_{h} S^{*} I^{*}}{1+\alpha I}\right)\left(1-\frac{I^{*}}{I}\right)\left(\frac{S I}{S^{*} I^{*}}-\frac{I}{I^{*}}\right)+\left(1-c_{1}\right) \frac{\beta_{e} S^{*} B^{*}}{B^{*}+\omega}\left(1-\frac{B^{*}}{B}\right)\left(\frac{I}{I^{*}}-\frac{B}{B^{*}}\right) .
\end{array}\right.
$$

Considering the function $V X=(V)=1-X+\ln X$, we know that $X>0$ which leads to $V(X) \leq 0$ and gives

$$
\begin{aligned}
& \left(1-c_{1}\right) \frac{\beta_{e} S^{*} B^{*}}{B^{*}+\omega}\left(1-\frac{S B\left(B^{*}+\omega\right)}{S^{*} B^{*}\left(B^{*}+\omega\right)}\right)+\left(1-c_{1}\right) \beta_{e} S^{*} \frac{B^{*}}{B^{*}+\omega}\left(1-\frac{I^{*}}{I}\right)\left(\frac{S\left(\beta_{e} S B /(B+\omega)\right)}{S^{*}\left(\beta_{e} S^{*} B^{*} /\left(B^{*}+\omega\right)\right)}-\frac{I}{I^{*}}\right) \\
& =\left\{\left(1-c_{1}\right) \beta_{e} S^{*} \frac{B^{*}}{B^{*}+\omega}\left\{1-\frac{S^{*}}{S}-\frac{S B\left(B^{*}+\omega\right)}{S^{*} B^{*}\left(B^{*}+\omega\right)}+\frac{S^{*} S B\left(B^{*}+\omega\right)}{S S^{*} B^{*}\left(B^{*}+\omega\right)}+\frac{S B\left(B^{*}+\omega\right)}{S^{*} B^{*}(B+\omega)}-\frac{I}{I^{*}}-\frac{I^{*} S B\left(B^{*}+\omega\right)}{I S^{*} B^{*}(B+\omega)}+1\right\}\right\} \\
& \leq\left\{\left(1-c_{1}\right) \beta_{e} S^{*} \frac{B^{*}}{B^{*}+\omega}\left\{\frac{B}{B^{*}}-\ln \left(\frac{B}{B^{*}}\right)-\frac{I}{I^{*}}+\ln \left(\frac{I}{I^{*}}\right)\right\}\right.
\end{aligned}
$$

Also,

$$
\begin{aligned}
& \left(1-c_{1}\right) \beta_{h} S^{*} I^{*}\left(1-\frac{S^{*}}{S}\right)\left(1-\frac{S I}{S^{*} I^{*}}\right)+\beta_{h} S^{*} I^{*}\left(1-\frac{I^{*}}{I}\right)\left(\frac{S I}{S^{*} I^{*}}-\frac{I}{I^{*}}\right) \\
& =\left(1-c_{1}\right) \beta_{h} S^{*} I^{*}\left(1-\frac{S I}{S^{*} I^{*}}-\frac{S^{*}}{S}+\frac{S^{*} S I}{S S^{*} I^{*}}+\frac{S I}{S^{*} I^{*}}-\frac{I}{I^{*}}-\frac{I^{*} S I}{I S^{*} I^{*}}+1\right) \leq 0 .
\end{aligned}
$$

Furthermore,

$$
\begin{aligned}
\left(1-c_{1}\right) \frac{\beta_{e} S^{*} B^{*}}{B^{*}+\omega}\left(1-\frac{B^{*}}{B}\right)\left(\frac{I}{I^{*}}-\frac{B}{B^{*}}\right) & =\left(1-c_{1}\right) \frac{\beta_{e} S^{*} B^{*}}{B^{*}+\omega}\left(\frac{I}{I^{*}}-\frac{B^{*} I}{B I^{*}}-\frac{B}{B^{*}}+1\right) \\
& \leq\left(1-c_{1}\right) \frac{\beta_{e} S^{*} B^{*}}{B^{*}+\omega}\left[\frac{I}{I^{*}}-\ln \left(\frac{I}{I^{*}}\right)-\frac{B}{B^{*}}+\ln \left(\frac{B}{B^{*}}\right)\right] .
\end{aligned}
$$

Therefore

$$
\begin{aligned}
\dot{V}= & \left\{\left(1-c_{1}\right) \frac{\beta_{e} S^{*} B^{*}}{B^{*}+\omega}\left[\frac{B}{B^{*}}-\ln \left(\frac{B}{B^{*}}\right)-\frac{I}{I^{*}}+\ln \left(\frac{I}{I^{*}}\right)\right]\right. \\
& +\left(1-c_{1}\right) \frac{\beta_{e} S^{*} B^{*}}{B^{*}+\omega}\left[\frac{I}{I^{*}}-\ln \left(\frac{I}{I^{*}}\right)-\frac{B}{B^{*}}+\ln \left(\frac{B}{B^{*}}\right)\right]=0
\end{aligned}
$$

Hence, the largest invariant subset at $\dot{V}=0$ is $\mathscr{E}^{*}$. Therefore, by La Salle's invariance principle, $\mathscr{E}^{*}$ is globally asymptotically stable when $R_{0}>1$ [33].

3.8. Model Fitting Technique. Analysing infectious disease data is a nonstandard problem. As a result, many approaches have been developed. This section takes into account the nonlinear least square method also known as 
the Nelder-Mead method, a mathematical technique, which is used to estimate the value of the parameters used in the cholera model. In order to implement the nonlinear least square method, the model system (1) is written in the following form:

$$
\left\{\begin{array}{l}
\frac{\mathrm{d}}{\mathrm{d} t} y(t, \theta)=f(t, y, \theta), \\
y(0, \theta)=y_{0}
\end{array}\right.
$$

where the function $f$ depends on time $t$ and the state variable $y$ and the vector of parameters $\theta$ are to be estimated. The vector of the state variable is given as

$$
y(t, \theta)=(S(t), I(t), R(t), B(t)) \in R^{4} .
$$

The idea used to perform the least square method for estimating parameters is to minimize the sum of difference between the observed data points $y\left(t_{i}\right)$ and the solution of the model $\hat{y}\left(t_{i}, \theta\right)$ associated with the model parameter $\theta$. Hence, given $n$ data points $\left(t_{i}, y_{i}\right)$, for $i=1, \ldots, n$, we seek to minimize the objective function given by

$$
M(\theta)=\sum_{i=1}^{n}\left[\widehat{y}\left(t_{i}, \theta\right)-y\left(t_{i}\right)\right]^{2} .
$$

Therefore, finding the parameter vector $\theta$, which best fits the model, implies solving the following optimization problem:

$$
\begin{array}{ll}
\min _{\theta} & M(\theta), \\
\text { subject to } & \theta_{\min } \leq \theta_{i} \leq \theta \max ,
\end{array}
$$

for $i=1, \ldots, m$, where $m$ is the number of parameters to be estimated. The model is implemented using Python and is then fit to real data obtained from the WHO data bank.

The control parameters can take a minimum value of 0 and a maximum value of 1 . If $\widehat{c_{1}}$ and $\widehat{c_{2}}<1$, then $c_{1}=c_{2}=0$, and if $\widehat{c_{1}}$ and $\widehat{c_{2}}>1$, then $c_{1}=c_{2}=1$. Otherwise, $c_{1}=\widehat{c_{1}}$ and $c_{2}=\widehat{c_{2}}$. Therefore, for the control parameters $c_{1}^{*}$ and $c_{2}^{*}$, the optimum value of the function $J\left(c_{1}, c_{2}\right)$ is obtained.

The rate of interactions and kinetics are estimated from real data derived from the World Health Organization Cholera data hub for Ghana [34]. We also consider published literature data where applicable. A suitable curve for our model as shown in Figure 2 is obtained using the data by applying the nonlinear least square (NLS) method as discussed above.

The control measures were used to determine the longterm effect of cholera using the data. From Figure 3, we observed the projected graph showing a decreasing trend of cholera cases when the controls are effectively applied. This decreasing trend may be due to the incorporation of education and treatment of water bodies in the model.

\section{Optimal Control Analysis}

In this section, we discussed the optimal control of the model by minimizing the spread of cholera using the control parameters $c_{1}$ and $c_{2}$. Let $c_{i}(t)=1$, for $i=1,2$, be linear

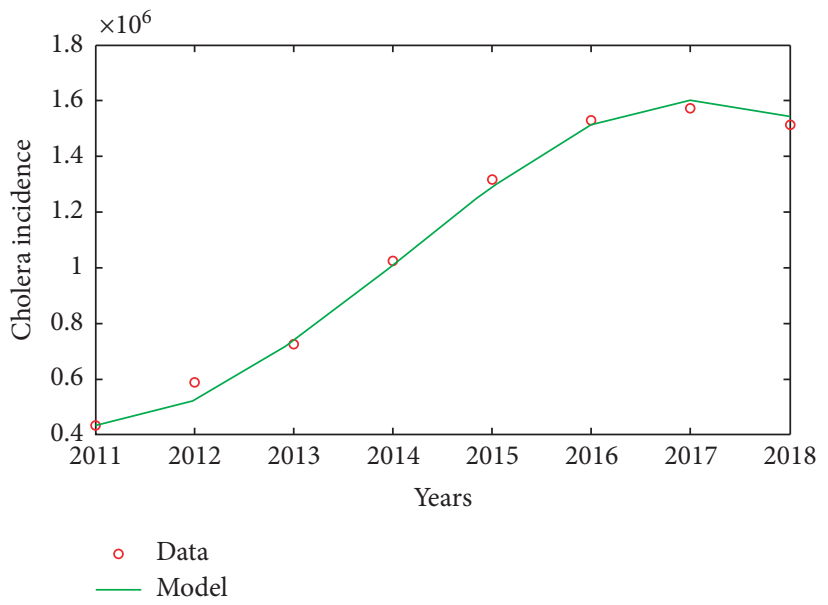

FIgURE 2: A plot showing the fitted model using the least square method for reported infected cholera cases.

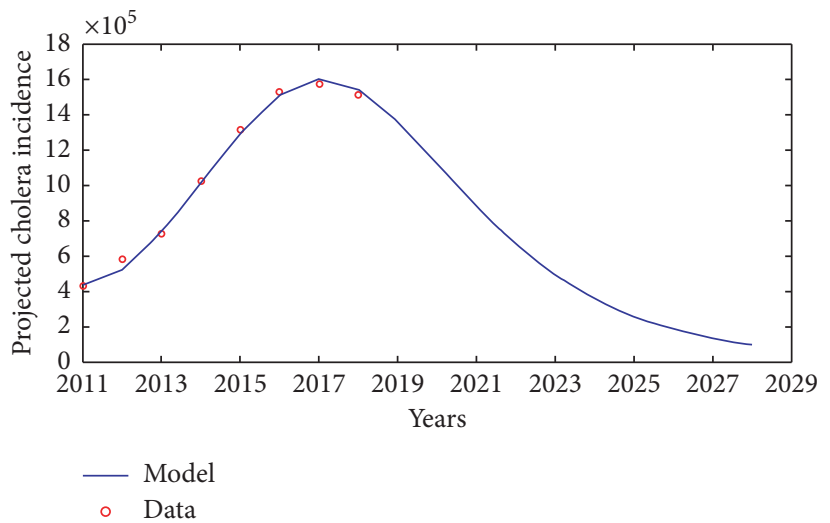

FIgURE 3: A plot showing the projection of cholera cases fitted to the actual data of the model. Parameters used are defined in Table 2.

functions. The control measures $c_{1}$ and $c_{2}$ are very effective when $c_{1}=c_{2}=1$ and not effective when $c_{1}$ and $c_{2}=0$. The control measure $c_{1}$ is the educational campaign given to individuals to help reduce cholera and $c_{2}$ is the treatment of water bodies. Considering the use of bounded Lebesgue measurable control, we define the objective function to be minimized as

$$
J\left(c_{1}, c_{2}\right)=\int_{0}^{t_{F}}\left(\psi_{1} I+\psi_{2} B+\frac{1}{2} a_{1} c_{1}^{2}+\frac{1}{2} a_{2} c_{2}^{2}\right) \mathrm{d} t,
$$

where $\left[0, t_{F}\right]$ is the time period of intervention. The dynamics of the controls to minimize the objective function is given by system (1). The associated basic reproduction number for system (1) is given in equation (17). We minimize the infectious human population $(I)$ and bacteria $(B)$, as well as the cost involved in implementing the control measures $c_{1}$ and $c_{2}$. The functional objective includes the social cost which relates to educating susceptible individuals $(1 / 2) a_{1} c_{1}^{2}$ and treatment of water bodies $(1 / 2) a_{2} c_{2}^{2}$. The quantity $\psi_{1}$ represents the cost associated with minimizing the infected human population, and $\psi_{2}$ represents the cost associated with minimizing the bacteria population in the 
environment. The costs corresponding to $\psi_{1} I$ and $\psi_{2} B$ are linear, whereas the cost control functions $(1 / 2) a_{1} c_{1}^{2}$ and $(1 / 2) a_{2} c_{2}^{2}$ are nonlinear. The Lagrangian of the optimal control problem is given by

$$
L=\left(\psi_{1} I+\psi_{2} B+\left(\frac{1}{2}\right) a_{1} c_{1}^{2}+\left(\frac{1}{2}\right) a_{2} c_{2}^{2}\right)
$$

To determine the Lagrangian minimum value, the Hamiltonian, $H$, for the control problem is defined as

$$
\begin{aligned}
H= & \psi_{1} I+\psi_{2} B+\left(\frac{1}{2}\right) a_{1} c_{1}^{2}+\left(\frac{1}{2}\right) a_{2} c_{2}^{2}+v_{S} \frac{\mathrm{d} S}{\mathrm{~d} t} \\
& +v_{I} \frac{\mathrm{d} I}{\mathrm{~d} t}+v_{R} \frac{\mathrm{d} R}{\mathrm{~d} t}+v_{B} \frac{\mathrm{d} B}{\mathrm{~d} t},
\end{aligned}
$$

where $v_{S}, v_{I}, v_{R}$, and $v_{B}$ are the adjoint variables. The differential equations of the adjoint variables are obtained by taking the partial derivatives of the Hamiltonian equation, that is, equation (49), with respect to the state variables which gives

$$
\begin{aligned}
& \left\{\begin{array}{l}
\frac{\mathrm{d} \nu_{S}}{\mathrm{~d} t}=\left(\nu_{S}-v_{I}\right)\left(\left(1-c_{1}\right)\left[\frac{\beta_{e} B}{B+\omega}+\frac{\beta_{h} I}{(1+\alpha I)}\right]\right)+v_{S}(\mu+\tau)-v_{R} \tau \\
\frac{\mathrm{d} \nu_{I}}{\mathrm{~d} t}=-\psi_{1}+\left[\left(1-c_{1}\right)\left(\frac{\beta_{h} S}{(1+\alpha I)^{2}}\right)\right]\left(\nu_{S}-\nu_{I}\right)+\nu_{I}(\mu+\gamma+\xi+\eta)-v_{R} \xi-v_{B} \eta \\
\frac{\mathrm{d} \nu_{R}}{\mathrm{~d} t}=v_{R} \mu \\
\frac{\mathrm{d} \nu_{B}}{\mathrm{~d} t}=-\psi_{2}+\left(\nu_{S}-v_{I}\right)\left(\left(1-c_{1}\right)\left(\frac{\beta_{e} S \omega}{(B+\omega)^{2}}\right)\right)+c_{2} \delta v_{B}-r \nu_{B}+\frac{2 r B K}{K^{2}} v_{B}-\psi_{2} .
\end{array}\right. \\
& \widehat{c_{1}}=\frac{\left(\nu_{I}-\nu_{S}\right)\left(\beta_{e} S B+\beta_{h} S I\right)}{a_{1}(B+\omega)(1+\alpha I)}, \\
& \widehat{c_{2}}=\frac{\nu_{B} \delta B}{a_{2}} .
\end{aligned}
$$

Theorem 6. Given the optimal controls $c_{1}$ and $c_{2}$ and the solutions $S, I, R$, and $B$ of corresponding state equation (50) which minimizes the objective function $J\left(c_{1}, c_{2}\right)$ over the region $\Omega$, then there exist adjoint variables $\nu_{S}, \nu_{I}, \nu_{R}$, and $\nu_{B}$ satisfying

$$
\frac{\mathrm{d} \nu_{i}}{\mathrm{~d} t}=-\frac{\partial H}{\partial i}, \quad i \in\{S, I, R, B\}
$$

Thus, the optimal solutions $c_{1}^{*}$ and $c_{2}^{*}$ are given by

$$
\begin{aligned}
& c_{1}^{*}=\min \left\{1, \max \left(0, \widehat{c_{1}}\right)\right\}, \\
& c_{2}^{*}=\min \left\{1, \max \left(0, \widehat{c_{2}}\right)\right\} .
\end{aligned}
$$

Proof. Differentiating the Hamiltonian with respect to the different control measures $c_{1}$ and $c_{2}$ and equating them to zero, we obtained

$$
\begin{aligned}
& \frac{\partial H}{\partial c_{1}}=a_{1} c_{1}+\left(\nu_{I}-v_{S}\right)\left(\frac{\beta_{e} S B}{B+\omega}+\frac{\beta_{h} S I}{1+\alpha I}\right)=0, \\
& \frac{\partial H}{\partial c_{2}}=-a_{2} c_{2}+\nu_{B} \delta B=0 .
\end{aligned}
$$

Making $c_{1}$ and $c_{2}$ the subject from equations (53) and (54), respectively, gives
Therefore, $\widehat{c_{1}}$ and $\widehat{c_{2}}$ are used in Python programming to make simulations.

4.1. Optimal Control Simulations. The Python programming language was used to simulate the optimal control model using the set of parameters obtained from the datasets. Some of these parameters are estimated for the sake of illustrations. Table 2 represents the values of the model parameters used for the simulations. The following initial conditions were also considered:

$$
\begin{aligned}
S & =60, \\
I & =50, \\
R & =0, \\
B & =0, \\
M_{1} & =1, \\
M_{2} & =1, \\
a_{1} & =5, \\
a_{2} & =10 .
\end{aligned}
$$


TABLE 2: The parameter values that give the best fit to the data in the model.

\begin{tabular}{lcc}
\hline Parameter & Value & Reference \\
\hline$\Delta$ & $0.03 /$ day & {$[20]$} \\
$\beta_{e}$ & $0.091371323 /$ day & Estimated \\
$\omega$ & $0.878455198 /$ day & Estimated \\
$\beta_{h}$ & $0.56341910 /$ day & {$[20]$} \\
$\alpha$ & $1.75189067 /$ day & Estimated \\
$\tau$ & $0.46791193 /$ day & Estimated \\
$\mu$ & $0.000048 /$ day & Estimated \\
$\gamma$ & $0.00132473 /$ day & Estimated \\
$\xi$ & $0.00116620 /$ day & Estimated \\
$K$ & 100000 cells/ml & {$[35]$} \\
$\eta$ & $0.001 /$ day & Assumed \\
$r$ & $0.03 /$ day & {$[20]$} \\
$\delta$ & $0.27707643 /$ day & Estimated \\
\hline
\end{tabular}

We described the controls using the following strategies (I, II, and III). However, Figures 4-6 represent the control profiles while the rest of the plots are the graphs of infectious human population and bacteria population plotted against time in years. They represent the effect of the optimal controls $c_{1}$ and $c_{2}$ in reducing the number of infected individuals as well as the bacteria in the environment.

\subsubsection{Strategy I: Control with Educational Campaign and} Treatment of Water Bodies. The strategy applied is to obtain the optimal control simulations that describes the effectiveness of the two control measures, that is, $c_{1} \neq 0$ and $c_{2} \neq 0$, when applied on the infectious class. The basic reproduction number obtained by applying this strategy is given by $R_{0}^{c}=0.4470$.

(1) Bacteria Population. When no control measures are applied, that is, $c_{1}=c_{2}=0$, the bacteria population is observed to increase. Access to safe drinking water and sanitation is very critical when it comes to the transmission of cholera. Therefore, when no control measure is being applied, the disease becomes persistent in the population. In the presence of control measures; that is, $c_{1} \neq 0$ and $c_{2} \neq 0$, the bacteria population in the environment decreases. Because treatment of water bodies minimizes the concentration of the bacteria in the population, individuals thus get access to safe drinking water. Also educational campaign creates the awareness for infected individuals more especially not to spread the disease since the purpose of educational campaign strategy is to explore the awareness of the disease, mode of transmission, and prevention. This therefore reduces the bacteria population in the environment as shown in Figure 7.

(2) Infected Individuals. When no control measure is applied, the number of infected individuals will increase in the population. On the other hand, when control measures are applied such as educational campaign, infected individuals would know the causes, transmission, and effects of cholera. This would reduce their actions which target environmental conditions that spread cholera. These activities

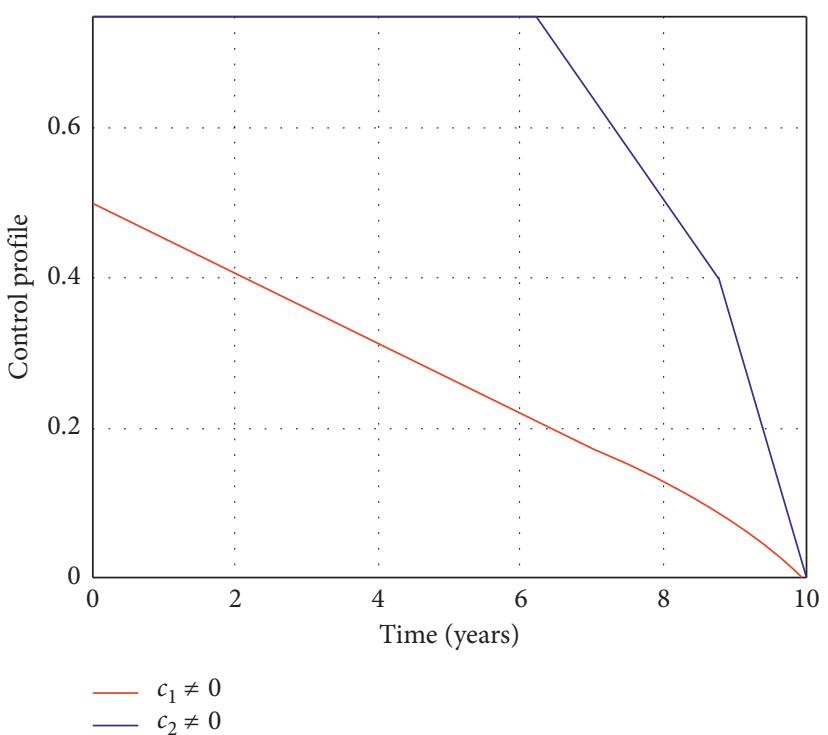

Figure 4: Control profile when $c_{1} \neq 0$ and $c_{2} \neq 0$ at $R_{0}^{c}=0.4470$.

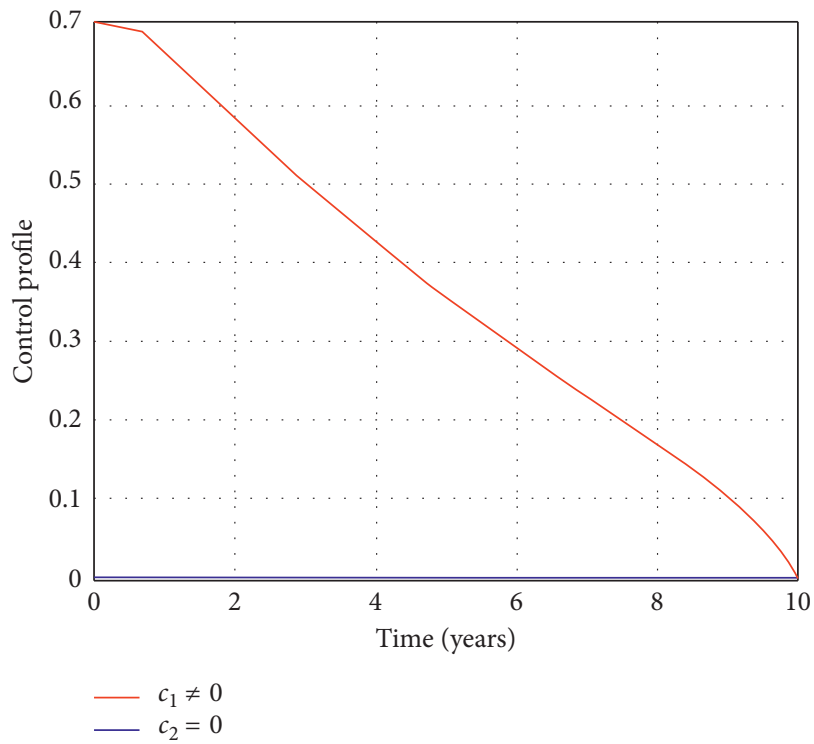

Figure 5: Control profile when $c_{1} \neq 0$ and $c_{2}=0$ at $R_{0}^{c_{1}}=0.6705$.

may include disruption of water bodies by defecating near water banks, individuals littering around causing bad sanitation systems, individuals crowding up in camps, etc. Hence, the number of infected individuals in the population would decrease as shown in Figure 8. On the other hand, when water bodies are treated, the rate of being reinfected decreases.

4.1.2. Strategy II: Control with Educational Campaign Only. The strategy applied is to obtain the optimal control simulations that describe the effectiveness of one control measure (that is, educational campaign) when applied. The basic reproduction number obtained by applying this strategy is given by $R_{0}^{c_{1}}=0.6705$. 


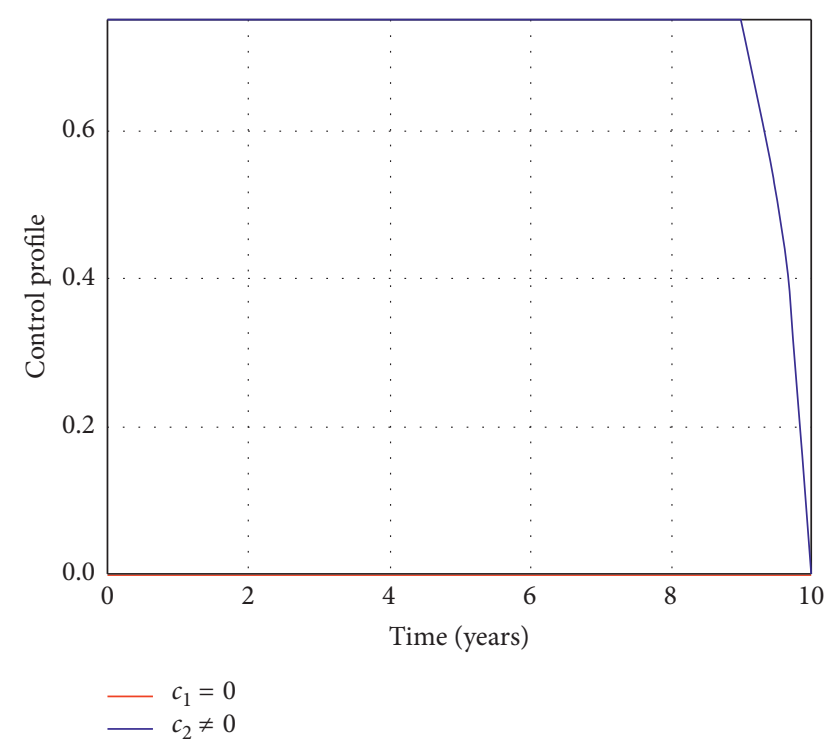

Figure 6: Control profile when $c_{1}=0$ and $c_{2} \neq 0$ at $R_{0}^{c_{2}}=2.2353$.

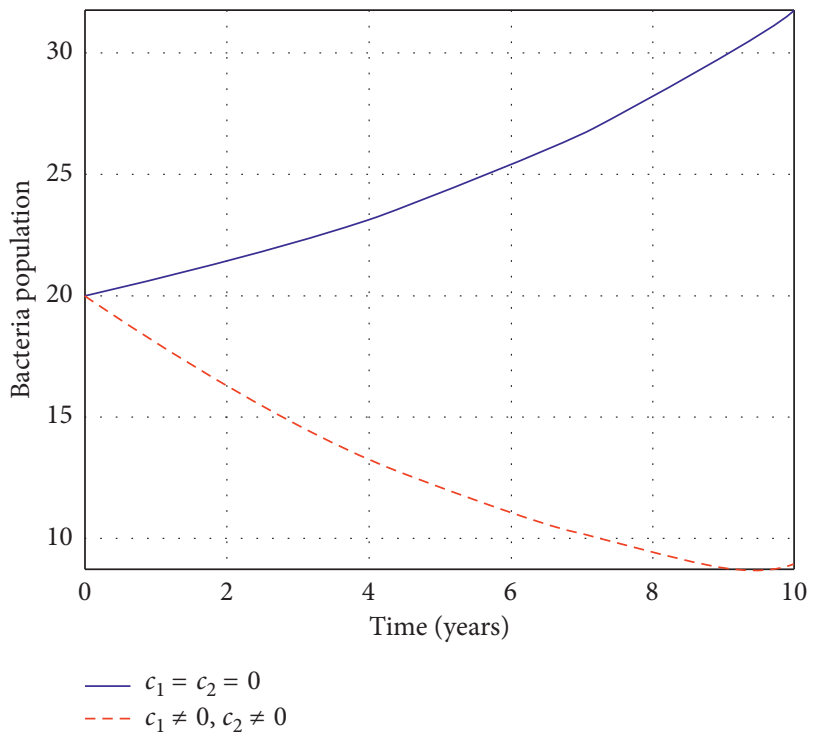

Figure 7: A graph showing the effectiveness of the two control measures $c_{1}$ and $c_{2}$ in the bacteria population to check its effect on the spread of cholera.

(1) Bacteria Population. When there are no control measures being applied, the bacteria population increases in the environment. Community engagement helps in the control of cholera. When individuals are educated by practically showing individuals how and the need to wash one's hands with soap and running water after visiting the toilet, it will reduce the bacteria they pick up from such areas. This helps reduce the bacteria population in the environment as shown in Figure 9. Thus, applying just educational campaign as a control measure is effective in controlling the spread of cholera.

(2) Infected Individuals. Infected individuals increase when there are no control measures being applied. Meanwhile, when infected individuals who are major contributors of the

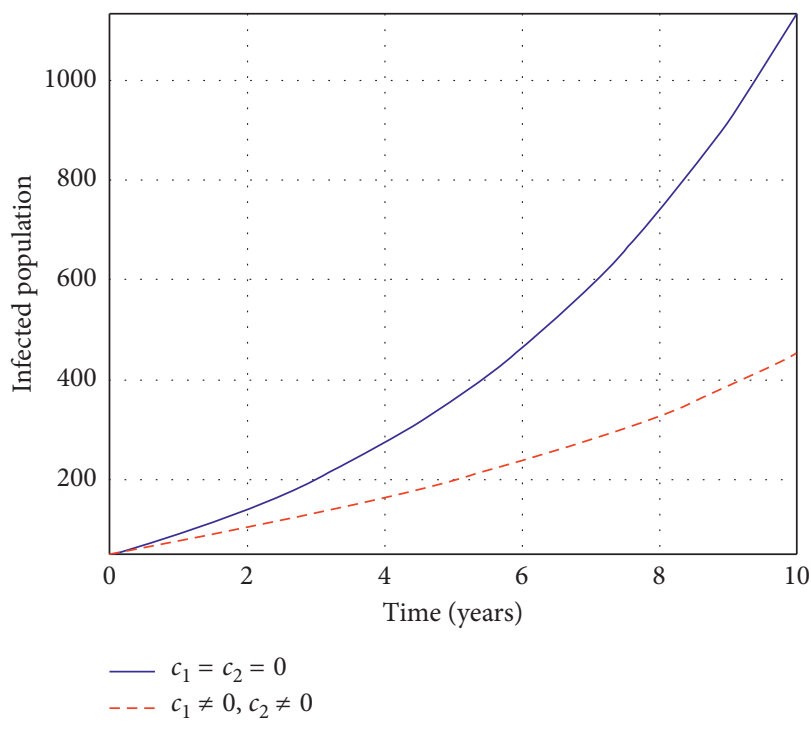

FIGURE 8: A graph showing the effectiveness of the two control measures $c_{1}$ and $c_{2}$ in the infected population to check its effect on the spread of cholera.

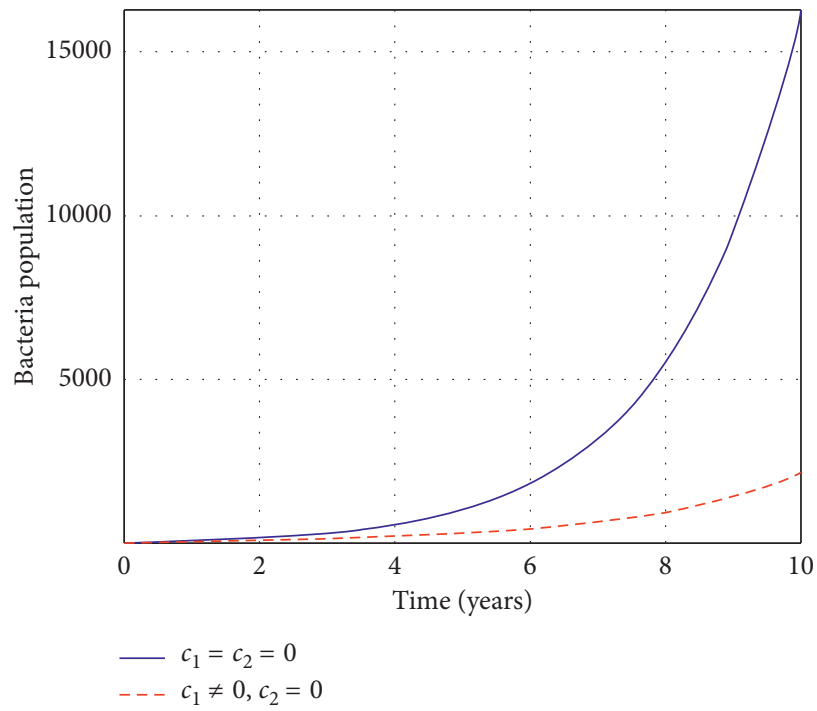

FIGURE 9: A graph showing the effectiveness of educational campaign as the only control measure to check its effect on the spread of cholera.

bacteria in the environment are educated on the causes, modes of transmission, and effect of cholera, their ignorant actions that spreads the bacteria would reduce. These individuals would know they have to defecate only at designated areas in order not to spread the bacteria. This goes hand in hand to help reduce the number of infected individuals in the population as shown in Figure 10. Hence, with the application of just one control measure, that is, by educating individuals, the spread of the disease can be controlled and is shown mathematically with an $R_{0}^{c_{1}}=0.6705$.

4.2. Strategy II: Control with Treatment of Water Bodies Only. Here, the strategy applied is to obtain the optimal control simulations that describe the effectiveness of treatment of 


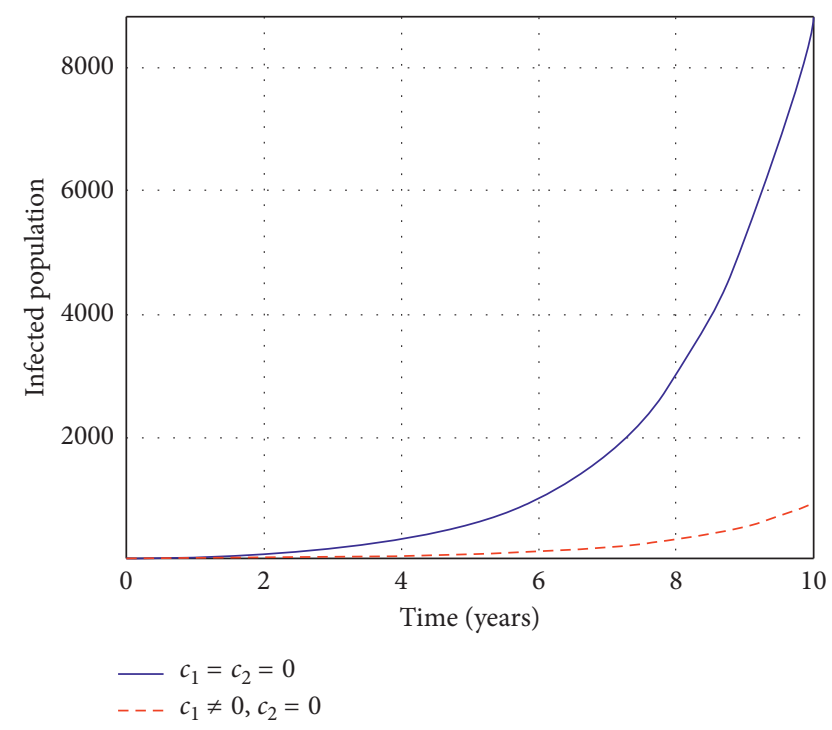

Figure 10: A graph showing the effectiveness of educational campaign as the only control measure $c_{1}$ in the infected population.

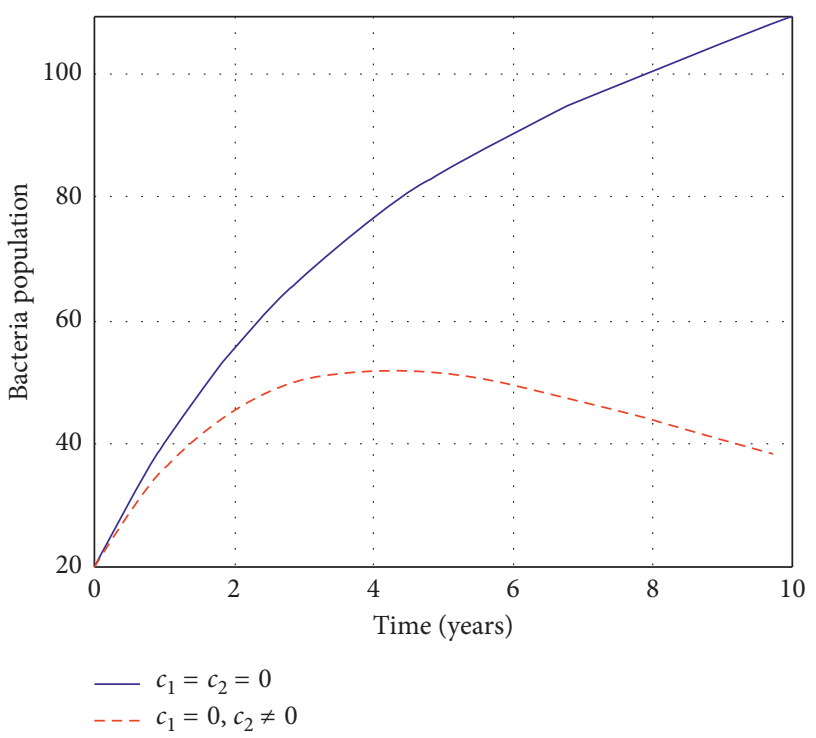

FIGURE 11: A graph showing the effectiveness of control measure $c_{2}$ only in the bacteria population.

water bodies as the only control measure being applied. The basic reproduction number obtained by applying this strategy is given by $R_{0}^{c_{2}}=2.2353$.

(1) Bacteria Population. The bacteria population in the environment increases when there are no control measures applied. However, when infected water bodies are treated, the bacteria population in the water body decreases due to the reduction in the concentration of the growth of the bacteria as shown in Figure 11.

(2) Infected Individuals. In Figure 12, it is observed that there is no change in the infected population even though the control (treatment of water bodies) is being applied. This is because in a wholly infected population, without a positive

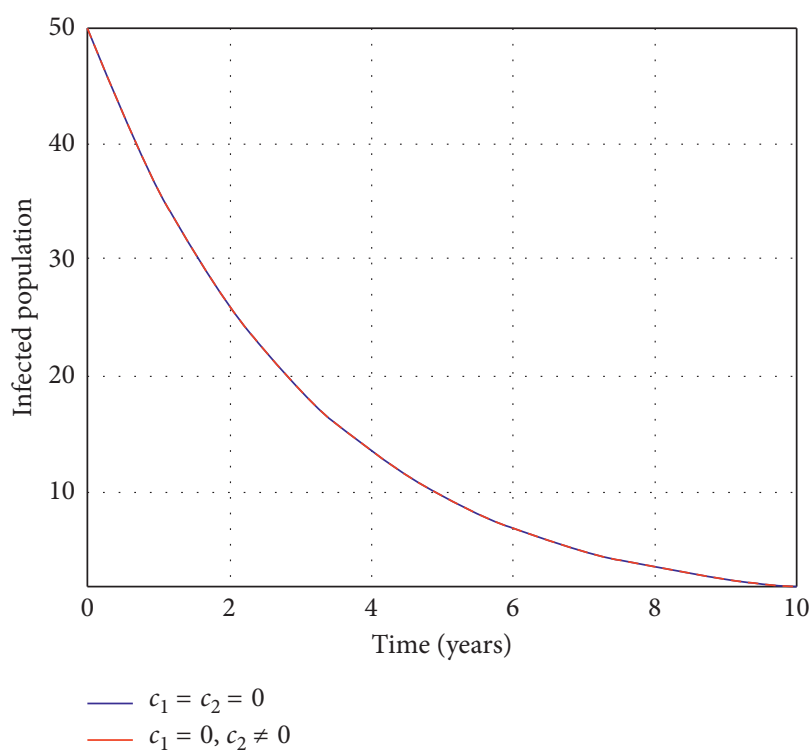

FIgURE 12: A graph showing the effectiveness of control measure $c_{2}$ only in the infected population.

modification of infected individuals behaviour, treatment of water bodies alone will yield no results. Hence, when infected water bodies are treated, it attains less effect on the infected individuals since there are no educational campaigns attached to help stop their ignorant activities that spread cholera. This is confirmed mathematical as the reproduction number assigned with treatment of water bodies as the only control measure in the population was observed to be greater than unity (i.e., $R_{o}^{c_{2}}=2.2353$ ).

\section{Conclusion}

In this paper, a mathematical model was developed and analysis was performed on the role of control measures and the environment in the transmission dynamics of cholera. The basic reproduction number was computed which plays the role of a threshold value for the dynamics of the system. The reproduction numbers with one or two control strategies were also derived. The local and global stabilities of both the disease-free equilibrium and endemic equilibrium were also proved. The model was optimized; educational campaign $c_{1}$ and treatment of water bodies $c_{2}$ as control measures were used to optimize the objective function defined by equation (47). The numerical simulations of the optimal control model show the effectiveness of these control measures when being applied. It was observed that the best alternative strategy when one control measure is to be applied is to educate individuals on the mode of transmission and prevention of cholera as against treatment of water bodies because a reproduction number of $R_{0}^{c_{1}}=0.6705$ was attained for the controlling of cholera through educational campaigns as against $R_{0}^{c_{2}}=2.2353$ for controlling of cholera through treatment of water bodies only. However, the best strategy is the application of both control measures, that is, applying both educational campaigns and treatment of water bodies. 


\section{Data Availability}

The data used to support the findings of this study were obtained from the World Health Organization data hub and referenced accordingly.

\section{Conflicts of Interest}

The authors declare that there are no conflicts of interest regarding the publication of this paper.

\section{Authors' Contributions}

All authors conceived and developed the study. All authors read and approved the final version of the manuscript.

\section{Acknowledgments}

The authors thank the support from the Next Einstein Initiative of the African Institute for Mathematical Sciences, Ghana (Annual Grant for Graduate Studies). The authors acknowledge, with thanks, the support of the African Institute for Mathematical Sciences, Ghana, for the production of this manuscript.

\section{References}

[1] A. McElroy and P. K. Townsend, Medical Anthropology in Ecological Perspective, Routledge, Abingdon, UK, 2004.

[2] J. M. Ochoche, "A mathematical model for the transmission dynamics of cholera with control strategy," International Journal of Science and Technology, vol. 2, no. 11, pp. 212-217, 2013.

[3] J. Sepúlveda, J. L. Valdespino, and L. García-García, "Cholera in Mexico: the paradoxical benefits of the last pandemic," International Journal of Infectious Diseases, vol. 10, no. 1, pp. 4-13, 2006.

[4] F. B. Osei and A. A. Duker, "Spatial dependency of V. cholera prevalence on open space refuse dumps in Kumasi, Ghana: a spatial statistical modelling," International Journal of Health Geographics, vol. 7, no. 1, p. 62, 2008.

[5] T. C. Matos, "Choleric fictions: epidemiology, medical authority, and an enemy of the people," Modern Drama, vol. 51, no. 3, pp. 353-368, 2008.

[6] S. Kanungo, B. K. Sah, A. L. Lopez et al., "Cholera in India: an analysis of reports, 1997-2006," Bulletin of the World Health Organization, vol. 88, no. 3, pp. 185-191, 2010.

[7] J. L. Deen, L. von Seidlein, D. Sur et al., "The high burden of cholera in children: comparison of incidence from endemic areas in Asia and Africa," PLoS Neglected Tropical Diseases, vol. 2, no. 2, p. e173, 2008.

[8] J. A. Al-Tawfiq, N. Andrews, A. Arguedas et al., "Extended list of references and summaries for the 2011 who position paper and the 2015 updated guidance on the use of meningococcal a conjugate vaccine," Morbidity and Mortality Weekly Report, vol. 60, pp. 1018-1019, 2011.

[9] J. Sharma, M. Malakar, M. Soni, and A. Pathak, "Outbreak of cholera in some villages of Boginodi area in Lakhimpur district of Assam," International Journal of Pharmacy and Biological Sciences, vol. 3, no. 3, pp. 450-454, 2013.

[10] R. Badkundri, V. Valbuena, S. Pinnamareddy, B. Cantrell, and J. Standeven, "Forecasting the 2017-2018 Yemen cholera outbreak with machine learning," 2019, https://arxiv.org/ftp/ arxiv/papers/1902/1902.06739.pdf.

[11] C. T. Codeço and F. C. Coelho, "Trends in cholera epidemiology," PLoS Medicine, vol. 3, no. 1, p. e42, 2006.

[12] J. G. Morris and D. Acheson, "Cholera and other types of vibriosis: a story of human pandemics and oysters on the half shell," Clinical Infectious Diseases, vol. 37, no. 2, pp. 272-280, 2003.

[13] M. Emch, C. Feldacker, M. S. Islam, and M. Ali, "Seasonality of cholera from 1974 to 2005: a review of global patterns," International Journal of Health Geographics, vol. 7, no. 1, p. 31, 2008.

[14] J. P. Tian, S. Liao, and J. Wang, "Dynamical analysis and control strategies in modeling cholera," 2010, http://www. math.ttu.edu/past/redraider2010/Tian2.pdf.

[15] C. T. Codeço, "Endemic and epidemic dynamics of cholera: the role of the aquatic reservoir," BMC Infectious Diseases, vol. 1 , no. 1, p. 1, 2001.

[16] R. Breban, J. M. Drake, and P. Rohani, “A general multi-strain model with environmental transmission: invasion conditions for the disease-free and endemic states," Journal of Theoretical Biology, vol. 264, no. 3, pp. 729-736, 2010.

[17] Z. Mukandavire, S. Liao, J. Wang, H. Gaff, D. L. Smith, and J. G. Morris, "Estimating the reproductive numbers for the 2008-2009 cholera outbreaks in Zimbabwe," Proceedings of the National Academy of Sciences, vol. 108, no. 21, pp. 8767-8772, 2011.

[18] A. R. Tuite, J. Tien, M. Eisenberg, D. J. D. Earn, J. Ma, and D. N. Fisman, "Cholera epidemic in Haiti, 2010: using a transmission model to explain spatial spread of disease and identify optimal control interventions," Annals of Internal Medicine, vol. 154, no. 9, pp. 593-601, 2011.

[19] G.-Q. Sun, J.-H. Xie, S.-H. Huang, Z. Jin, M.-T. Li, and L. Liu, "Transmission dynamics of cholera: mathematical modeling and control strategies," Communications in Nonlinear Science and Numerical Simulation, vol. 45, pp. 235-244, 2017.

[20] N. Ainea, A. Matofali, and M. Mkwizu, "Optimal control analysis of a cholera disease transmission model in Tanzania," The International Journal of Innovative Research in Science, Engineering and Technology, vol. 4, no. 4, pp. 865-872, 2019.

[21] N. J. Ezeagu, H. A. Togbenon, and E. Moyo, "Modeling and analysis of cholera dynamics with vaccination," American Journal of Applied Mathematics and Statistics, vol. 7, no. 1, pp. 1-8, 2019.

[22] F. B. Agusto and M. A. Khan, "Optimal control strategies for dengue transmission in Pakistan," Mathematical Biosciences, vol. 305, pp. 102-121, 2018.

[23] M. A. Khan, S. W. Shah, S. Ullah, and J. F. Gómez-Aguilar, “A dynamical model of asymptomatic carrier zika virus with optimal control strategies," Nonlinear Analysis: Real World Applications, vol. 50, pp. 144-170, 2019.

[24] M. A. Khan, A. Ali, L. C. C. Dennis et al., "Dynamical behavior of cholera epidemic model with non-linear incidence rate," Applied Mathematical Sciences, vol. 9, no. 20, pp. 989-1002, 2015.

[25] K. O. Okosun, M. A. Khan, E. Bonyah, and O. O. Okosun, "Cholera-schistosomiasis coinfection dynamics," Optimal Control Applications and Methods, vol. 40, no. 4, pp. 703-727, 2019.

[26] S. Ullah, M. A. Khan, and J. F. Gómez-Aguilar, "Mathematical formulation of hepatitis B virus with optimal control analysis," Optimal Control Applications and Methods, vol. 40, no. 3, pp. 529-544, 2019. 
[27] D. T. Leung, T. Uddin, P. Xu et al., "Immune responses to the o-specific polysaccharide antigen in children who received a killed oral cholera vaccine compared to responses following natural cholera infection in Bangladesh," Clinical and Vaccine Immunology, vol. 20, no. 6, pp. 780-788, 2013.

[28] O. Diekmann, J. A. P. Heesterbeek, and J. A. J. Metz, "On the definition and the computation of the basic reproduction ratio $R_{0}$ in models for infectious diseases in heterogeneous populations," Journal of Mathematical Biology, vol. 28, no. 4, pp. 365-382, 1990.

[29] P. van den Driessche and W. James, "Further notes on the basic reproduction number," in Mathematical Epidemiology, pp. 159-178, Springer, Berlin, Germany, 2008.

[30] P. K. Maini and A. Korobeinikov, "A Lyapunov function and global properties for sir and seir epidemiological models with nonlinear incidence," Mathematical Biosciences and Engineering, vol. 1, no. 1, pp. 57-60, 2004.

[31] H. E. Gervas, N. K.-D. O. Opoku, and S. Ibrahim, "Mathematical modelling of human African trypanosomiasis using control measures," Computational and Mathematical Methods in Medicine, vol. 2018, Article ID 5293568, 13 pages, 2018.

[32] S. Olaniyi, M. A. Lawal, and O. S. Obabiyi, "Stability and sensitivity analysis of a deterministic epidemiological model with pseudo-recovery," IAENG International Journal of Applied Mathematics, vol. 46, no. 2, pp. 1-8, 2016.

[33] J. Hespanha, "Uniform stability of switched linear systems: extensions of La Salle's invariance principle," IEEE Transactions on Automatic Control, vol. 49, no. 4, pp. 470-482, 2004.

[34] WHO, "Global health observatory data repository: number of reported cases data by country," 2018, http://apps.who.int/ gho/data/node.main.175.

[35] S. Edward and N. Nyerere, "A mathematical model for the dynamics of cholera with control measures," Applied and Computational Mathematics, vol. 4, no. 2, pp. 53-63, 2015. 